\title{
Do genes affect morphine response?
}

\author{
Louise Ladebo ${ }^{1}$ \& Anne Estrup Olesen *,1,2 \\ ${ }^{1}$ Mech-Sense, Department of Gastroenterology \& Hepatology, Aalborg University Hospital, Aalborg \& Clinical Institute, Aalborg \\ University, Denmark \\ ${ }^{2}$ Department of Drug Design \& Pharmacology, Faculty of Health \& Medical Sciences, University of Copenhagen, Copenhagen, \\ Denmark \\ * Author for correspondence: Tel.: +45 9766 0535; aneso@rn.dk
}
"different haplotypes and combinations of genetic variants can affect protein function synergistically and thus alter morphine response substantially."

First draft submitted: 8 August 2017; Accepted for publication: 8 August 2017; Published online: 24 October 2017

Keywords: analgesia $\bullet$ morphine $\bullet$ pharmacodynamics $\bullet$ pharmacogenetics

Morphine is an essential medication for treatment of severe acute and chronic pain. However, interindividual differences in pain sensitivity and analgesic response make the clinical use of morphine challenging. Approximately $10-30 \%$ of patients report an unsatisfactory pain relief or intolerable side effects, which often leads to drug withdrawal. Several confounding factors such as age, gender, mental state, polypharmacy, organ function, comorbidity and certain lifestyle habits can influence the therapeutic outcome and thus contribute to the variability seen. Genetic variation has also shown to alter the pharmacokinetics and pharmacodynamics of morphine. In the following, a brief overview will be provided on how genes encoding pharmacokinetic (drug transporters or metabolizing enzymes) and pharmacodynamic (receptors or enzymes) factors can play a vital role for the efficacy of morphine.

\section{Polymorphisms affecting drug transporters}

Genetic polymorphisms can alter the functionality of gene products and thus affect drug response and severity of side effects. Morphine is a substrate for P-gp, which is an efflux transporter, belonging to the ATP-binding cassette $(\mathrm{ABC})$ family, encoded by the $A B C B 1$ gene. P-gp serves as a defender against xenobiotics by limiting drug absorption over the small intestine and facilitating excretion by transporting drugs into bile - and urine ducts. Last but not least, it also transports xenobiotics out of the brain across the blood-brain barrier, thereby minimizing drug-receptor interactions.

In vitro studies have found an association between polymorphisms of the $A B C B 1$ gene and lower glycoprotein activity and expression levels in the duodenum, natural killer cells and renal parenchyma [1]. Some observational studies in cancer patients have also found an association between $A B C B 1$ polymorphisms and altered analgesic responses and dose requirements. However, other similar study approaches reveal no such relationship [2,3]. Moreover, no significant results have been found in human experimental pain models and controlled clinical-pain models $[1,2,4]$. This could be due to poor study designs lacking relevant end points (e.g., side effects and pharmacodynamic response). Presently, the C3435T polymorphism is the most studied $A B C B 1$ SNP. It is plausible that other $A B C B 1$ SNPs have a clinical significance, although additional studies are needed to confirm this hypothesis.

Another but functionally different transporter is the OCT1, which transports organic cations such as morphine into hepatocytes, thus potentially influencing morphine disposition and hepatic metabolism. Currently, no strong evidence suggests that the OCT1 reduced-function polymorphisms affect morphine response. However, one study did find a significant higher morphine $\mathrm{AUC}_{0-24 \mathrm{~h}}$ and an insignificant higher $\mathrm{C}_{\max }$ in healthy Caucasian carriers. Unfortunately, no pharmacodynamic end points were evaluated, wthereby the clinical impact is impossible to discuss [5]. 


\section{Polymorphisms affecting drug metabolism}

Morphine is primarily metabolized in the liver by UGT2B7 to morphine-3-glucuronide (90\%) and morphine6-glucuronide (10\%). Thus, increased activity of UGT2B7 can result in lower morphine concentrations and subsequently reduced analgesia. On the other hand, decreased activity can lead to higher morphine concentrations with an increased effect and risk of severe side effects. A limited number of trials have investigated the effects of $U G T 2 B 7$ variants on glucuronidation activity and results are inconsistent across different study approaches $[1,3]$. Thus, the clinical impact of $U G T 2 B 7$ variants is debatable and further exploration is necessary.

A minor percentage of morphine is metabolised by CYP enzymes. Few human postoperative pain studies have shown that polymorphisms in CYP genes influence morphine response and drug consumption. However, reports from human experimental pain studies fail to confirm any association and to our knowledge no observations from disease states exist [2]. CYP polymorphisms may not be clinically important due to their scarce role in morphine metabolism. This may also explain the small number of studies performed so far.

\section{Polymorphisms affecting pharmacodynamic factors}

Polymorphisms of genes coding receptor functions can alter drug affinity, sensitivity and specificity, thereby affecting drug potency and efficacy. Morphine exerts its analgesic effect mainly by binding to the $\mu$-opioid receptor found in the central nervous system. The $\mu$-opioid receptor is encoded by the OPRM1 gene and the most commonly studied SNP is the A118G nucleotide substitution. Studies in cancer and postoperative patients show that carriers of the homozygote $G$ allele have higher pain scores and require higher morphine doses, thereby indicating a reduced signaling efficacy and possibly declined receptor expression [2,3]. Yet again, results from other similar studies are contradictory and human experimental studies are lacking. The significance of other opioid receptor polymorphisms (e.g., OPRK1 and OPRD1) may also be relevant to look closer into, as morphine partly interacts with these receptors also.

COMT is an enzyme that modulates the neurotransmission of dopamine and norepinephrine. Pain studies in postoperative and cancer patients have demonstrated conflicting results regarding an association between polymorphisms in the COMT gene and morphine response [2]. However, a recent postoperative study showed that combinations of genetic variants for example OPRM1 and COMT better explained variability in morphine consumption than single genetic variants [6. Previous studies have also reported an association between COMT haplotypes and altered pain sensitivity [7]. Hence, different haplotypes and combinations of genetic variants can affect protein function synergistically and thus alter morphine response substantially.

In general, additional human studies are needed to further elucidate the significance of genetic variability on morphine efficacy. These should: genotype and determine expression levels of numerous candidate genes and associated SNPs; include combinations of alleles (haplotypes); be large scale studies to avoid underpowered results with low frequencies of the explored SNPs; include different types of pain modalities and side-effect scorings for better pharmacodynamic evaluation; include frequent blood sampling for improved pharmacokinetic evaluation; be systematically designed with specific in- and exclusion criteria, so confounding factors are minimized as much as possible.

\section{Confounding factors}

It is well-known that our kidney and liver function gradually worsens as we age. Consequently, this affects drug metabolism and excretion with a subsequent amplified analgesic response and/or severity of side effects. Since the majority of morphine-users are adults and elderly, it is possible that part of the interindividual variability in response to morphine is correlated to the processes associated with aging. Hypothetically, genetic variability may therefore influence pharmacokinetic factors to a larger degree in young individuals compared with elderly, where the physical condition of the patient and lifestyle factors may be superior.

A person's lifestyle may also considerably alter the response to morphine. Co-administration of some mechanistically similar drugs has for example resulted in additive effects and additional adverse events [8]. Certain drugs and foods, cigarettes and alcohol can also potentially up- and down-regulate the expression of pharmacologically important gene products. Hence, interaction studies with morphine are warranted.

Pain perception and sensitivity is very subjective and influenced by cognitive disturbances and impairments, diseases, fear and anxiety. Thus, the interindividual response to analgesics is also confounded by psychological factors, which should be included as covariates in future clinical studies. 


\section{Conclusion}

Different genetic polymorphisms may affect morphine response. However, the current conflicting evidence makes it difficult to conclude the magnitude of specific gene variants. Nevertheless, interindividual variability is most likely not due to a single SNP, but rather several genetic variants and/or nongenetic factors being present simultaneously, thereby creating a synergistic effect. Further research is necessary and this will undoubtedly enhance our understanding of how genetic differences contribute to the complex mechanisms and interactions during pain and its treatment.

\section{Financial \& competing interests disclosure}

The authors have no relevant affiliations or financial involvement with any organization or entity with a financial interest in or financial conflict with the subject matter or materials discussed in the manuscript. This includes employment, consultancies, honoraria, stock ownership or options, expert testimony, grants or patents received or pending, or royalties.

No writing assistance was utilized in the production of this manuscript.

\section{References}

1 Nielsen LM, Sverrisdóttir E, Stage TB et al. Lack of genetic association between OCT1, ABCB1 and UGT2B7 variants and morphine pharmacokinetics. Eur. J. Pharm. Sci. 99(99), 337-342 (2017).

2 Nielsen LM, Olesen AE, Branford R, Christrup LL, Sato H, Drewes AM. Association between human pain-related genotypes and variability in opioid analgesia: an updated review. Pain Pract. 15(6), 580-594 (2015).

3 Baber M, Bapat P, Nichol G, Koren G. The pharmacogenetics of opioid therapy in the management of postpartum pain: a systematic review. Pharmacogenomics 17(1), 75-93 (2016).

4 Bastami S, Gupta A, Zackrisson A-L, Ahlner J, Osman A, Uppugunduri S. Influence of UGT2B7, OPRM1 and ABCB1 gene polymorphisms on postoperative morphine consumption. Basic Clin. Pharmacol. Toxicol. 115(5), 423-431 (2014).

5 Tzvetkov MV, dos Santos Pereira JN, Meineke I, Saadatmand AR, Stingl JC, Brockmöller J. Morphine is a substrate of the organic cation transporter OCT1 and polymorphisms in OCT1 gene affect morphine pharmacokinetics after codeine administration. Biochem. Pharmacol. 86(5), 666-679 (2013).

6 De Gregori M, Diatchenko L, Ingelmo PM et al. Human genetic variability contributes to postoperative morphine consumption. J. Pain. 17(5), 628-636 (2016).

7 Diatchenko L, Slade GD, Nackley AG et al. Genetic basis for individual variations in pain perception and the development of a chronic pain condition. Hum. Mol. Genet. 14(1), 135-143 (2005).

8 Kotlinska-Lemieszek A, Klepstad P, Faksvåg Haugen D. Clinically significant drug-drug interactions involving opioid analgesics used for pain treatment in patients with cancer: a systematic review. Drug Des. Devel. Ther. 16(9), 5255-5267 (2015). 
(

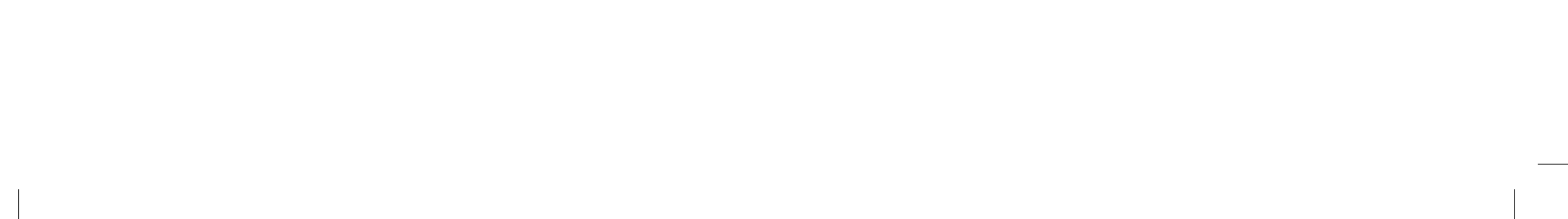

